\title{
Towards a Possible Entropy-Driven Process of Consciousness
}

\author{
Pierre R. Blanquet ${ }^{1,2}$ \\ ${ }^{1}$ Club d'Histoire des Neurosciences, Université Pierre et Marie Curie, Paris, France \\ ${ }^{2}$ Lab. SHERE, Université Paris-Diderot, Paris, France \\ Email: pr.blanquet@free.fr
}

How to cite this paper: Blanquet, P.R. (2018) Towards a Possible Entropy-Driven Process of Consciousness. Journal of Behavioral and Brain Science, 8, 674-696. https://doi.org/10.4236/jbbs.2018.812041

Received: November 21, 2018 Accepted: December 24, 2018 Published: December 27, 2018

Copyright (c) 2018 by author and Scientific Research Publishing Inc. This work is licensed under the Creative Commons Attribution International License (CC BY 4.0).

http://creativecommons.org/licenses/by/4.0/

\section{cc) (i) Open Access}

\begin{abstract}
In the present article, we argue that consciousness and body are not to be separate to explain the intentional consciousness of the human being facing to environment. 1) The most neurobiologist and neurocognitivist studies suggest that the emerging consciousness appears to be a generic recurring process which subsumes under it the various results of scientist approaches. When analyzed with the cybernetic transductive method of Simondon, this process can be viewed as a noetico-neuronal unit which the sui generis activity can be boiled down to concept of intentional consciousness. 2) Homeostasis can be also metaphorically described as a generic recurring process which subsumes under it the myriad molecular feedback loops of organism. This self-regulating dynamic process could be governed by the cosmologic (Earth + Universe) production of entropy, which would keep high activity and dissipating energy and low internal entropy to maintain an ordering in the organic structure. 3) Based on numerous arguments and data, the cerebral noetico-neuronal process and the self-regulating homeostasic process may be viewed as an entire physiological (brain + body) system. We suggest that the whole (brain + body) has an intentional embodied consciousness that could be driven by the cosmologic production of entropy ${ }^{1}$.
\end{abstract}

\section{Keywords}

Emerging Consciousness, Noetico-Neuronal Process, Intentional Embodied Consciousness, Physiological Homeostasis, Cosmologic Entropy Production

\section{Introduction}

Many current data suggest that consciousness and body are necessary sets to the

${ }^{1}$ This paper has been the subject of a conference on May 5, 2017 at the Paris-Diderot University in Paris. 
self identity and the homeostasic unit of the human being [1]-[6]. Without awareness, the body could not be voluntary, as evidenced by the neuro-vegetative coma, but without corporeity, consciousness could not be free. The self-maintaining of the subject requires us to believe that it is intentional psycho-physical unit that maintains the life of the individual. However, since the world is never outside the body, insofar as it belongs to the bodily system, we can admit that the human is always a whole "world-body-consciousness". So, in the light of this view, it should be possible to formulate a model of conscious psycho-physical unit whose intentionality would be due to interaction between two poles: a pole subject cognitive and a pole object "attractor". Recently, based on increasing evidence that the quantum physics is probably important for understanding of some biological phenomena, several neuro-scientists struggled to explain the permanent interaction between the brain and the environment in terms of process of quantum coherence [7]. Unfortunately, quantum coherence process in a wet biological system such as brain would seemingly require fulfillment of many unrealizable conditions. At the present time, there is impossibility to seriously propose a model of conscious psycho-physical unit which has only a quantum principle as a basis. Another possibility would be that the psycho-physical intricacy that keeps alive the individual is governed by the laws of dissipative nonequilibrium thermodynamic systems. As suggested by Nicolis and Prigogine [8], the alive being seems to behave as a non-isolated dissipative thermodynamic system which maintains its balance with the outside by pulling permanently energy from the environmental world. Such a mechanism allows the being to keep a usable cosmological entropy (and hence an order) in its physiological system. In this article, we show that interaction between the pole cognitive and the pole object could be actually of cosmological order, insofar as the pole "attractor" object could be likened to increasing entropy production of the world (Earth + Universe) $)^{2}$.

\section{Approaches to the Problem}

In the third-person perspective of objective consciousness, the majority of models of consciousness were elaborate with neurobiological, neurophysical and cognitive approaches. Philosophers and scientists who deal with the objective consciousness contents belong to two main streams of thought: dualism and monism [9]. Their efforts were intensified following espousal of dualism of Descartes and of monistic idealism of Berkeley. In the dualistic conceiving, the neural "substrate" is the transitive cause of the noetic "substrate". The transitive cause comes out of itself to produce an effect out of it. For example, in the crypto-dualistic model of Stuart Hameroff and Roger Penrose [9], consciousness happens when microtubules of some neurons produce a phase of quantum superposition (pre-conscious state) that reaches a non-computational threshold "In this article, the term of "noetic" will be used rather than the term of "psychic". Noetic means "relative to very act of consciousness". It is therefore more specific that the term of "psychic" which is relative to mind. 
called "objective reduction" by the authors. At this threshold occurs a sudden and discreet conscious event (an opportunity to experience, according to Whitehead [10]). One can also quote the models of Susan Pockett and Johnjoe MacFadden, based on the alleged action of a cortical electromagnetic field [7]. By contrast, in the monistic conceiving, neural "substrate" can be conceptually described as the emanative cause of the noetic "substrate": its effect is still out of itself, but it remains nevertheless in itself as a cause [11]. The origin of this design can be found in the metaphysics of light, mainly dating from the first century of our era with Pseudo-Dionysius of the Areopagus, in the fifth century with Augustin, and in the 13th century with Gosseteste. In the metaphysics of light, it was considered that it occurred at the same time the lux, the activity of divine, and the lumen, the radiation [12]. Except for the monism of Donald Davidson [13] on the philosophical level, the most active current contemporary researches based on a crypto-monistic conceiving usually use the neurobiological methodology. They are all based more or less explicitly on the assumption that mind transcends the organization and activity of a certain number of neuronal circuits while emanating from them. In these emergentist models, consciousness is suggested to be simultaneous to the neuronal activity: consciousness should be not caused, strictly speaking, by this activity, one and the other would constitute an unitary process.

From the second half of the 20th, a growing number of research suggested that body and consciousness could be modeled along the lines of nonlinear complex dynamical systems implying a noetico-bodily intricacy in the conscious cognition. The entirely of the Phenomenology of Perception of Maurice Merleau-Ponty, for example, was devoted to illustrating that the body cannot be viewed solely as an object or material entity of the world [14] [15]. This means that the perceiving human mind is an incarnated body: it enriches the concept of body to allow he both think and perceive. To Merleau-Ponty, it is for these reasons that we are best served by referring to the individual as not simply a body, but rather as a "body-subject". Proposals by Evan Thompson and Franscisco Varela [16] can be also cited to illustrate this. In an autopoietic framework describing the relationships between neuronal dynamics, consciousness, and embodiment, they proposed to investigate the neuronal correlates of consciousness. In doing so, they did not assume only a one-way causal relationship between neuronal systems and consciousness contents, but also the possibility of a two-way causal relationship between embodied conscious states and neuronal activities. In this view, the coupled dynamics of brain, body, and environment exhibits self-organization and emergent processes involving both upward and downward causality at multiple levels, including at the level of conscious acts in relation to neuronal activities. On the other hand, Edelman [5] [17] argued that a dynamic workspace core in brain may be sensitive to bodily (sensory) inputs or from other brain systems with which it interacts, as well as with itself. This gives rise to extremely complicated patterns of interactions in which the neuronal system is embodied. These dynamics, and especially the dynamic core hypothesis, 
are compatible with the idea that the brain is some kind of complex dynamical system. The theory of nonlinear complex dynamical systems could be thus a useful tool for this subject of study. In this view, Klaus Mainzer [18] [19] developed a theory characterized by non-linearity, in which non-linear equations, feedback loops and complex interactions can lead to auto-organizations of new structures. To Mainzer, such a complex dynamic system could be capable of modeling the neuronal interactions at the lower level, and consequently the emergence of macroscopic cognitive structures. From these data and hypothesis, several neuro-scientists suggested that free-will could be a nonlinear, chaotic and usually circular causality, resulting from complex interactions between the environmental context, the body and the consciousness [9].

More recently, cognitive science has insisted that consciousness is embodied, embedded, enacted, and that what now we call "the mind" does not cut off from our corporeal existence in the world and our interactions with it. As explained by Antonio Damasio, for example, brain makes charts of entries and reentries of signals that will tell it what's going on in the body and outside. If we observe indeed a piece of the cerebral cortex, one sees sets of lines constantly fluctuating. They result from the activity of neural networks at a given moment. Some networks draw the structure of the body, for example the level of looseness of muscles, others transmit sensory images. The mental images formed by these charts are then analyzed and used. However, it is not at the level of the cortex that the analysis of these images begins, but below, in the region of the brain called brainstem. This is the first step in a process that operates from the bottom up to the cortex [20]. Multi-disciplinary studies attempted also to show that brain is a part of our bodily experiments. For example, various approaches of psychotherapy were recently used to help explicate relationships between mind and body [21]. Particularly, the neuro-cognitivists Alain Berthoz and Jean-Luc Petit [2] [3] contributed to reconsider the body, the action and the cognition in human in the light of neurosciences. This physio-phenomenology of action now describes the brain like an externalisator structuring perception in the world by a series of perceptive assumptions. There is a dimension tensive, intentional, protensio-retentional of experiments of alive: brain continuously projects perceptions on the world as true tests of perceptive assumptions. Furthermore, it is necessary to suppose an intentional action of the body in the world. Indeed, it is the world which produces its consciousness in the body by starting the intentional operation of the bodily structure. In other words, body and world are not to be separated in a philosophy of consciousness. As Andrieu said, the brain is corporant by the indefinite incorporation of information, and the body is celebrating by their dynamic treatment [1]. Since we must recognize that consciousness is experienced in terms of dual relationship among the body and the brain, the difficulty is now to describe how the body produces the noetic meanings that are incorporated without reducing the noetic contents to physical data. The noetico-physical intricacy should describe how the interaction between the noetic activity and the body activity could generate an intentional act that constantly 
protects the unity of the individual facing to environment. More precisely, approach should describe how the noetico-body intricacy could ensure the self-maintaining of the individual in involving necessarily the emerging consciousness and the physiological homeostasis. Attempts to study this question should thus examine both the features of objective consciousness and the features of physiological homeostasis. At this point, let us recall that the main neurobiological and neurocognitive studies of objective consciousness relates mainly to four cerebral functional systems: the visual system, the olfactory system, the thalamocortical regions, and the neuronal systems seen from the point of view of the "cognitive reversal" [9] [22]. In the present article, we will first analyze the results of these emergentist scientific studies. We will then look at what characterizes the process of homeostasis.

\section{Neurobiological and Neurocognitivist Studies on Consciousness}

\subsection{Visual and Olfactory Systems [9]}

Studying visual system was mainly addressed by Viktor Lamme, Semir Zeki, David Milner and Melvyn Goodale. In his "Local recurrence theory", Lamme proposed that there is a main access path to consciousness. Initially, information from the retina and geniculate body is sent to the ridged visual area V1. It is then sent to extra-ridged areas as well as to parietal and temporal cortex. This process is not accompanied by a conscious experience. Information is then returned to the visual cortex V1 and produces the visual experience. The model also includes a process of recurring causation that extends towards the brain areas of action, language and attention. According to Zeki, there is multiple micro-consciousness of visual origin. In his model, visual areas specialized for color (V1, V2, V4), shape (V1, V2, V3, V4) and movement (V1, V2, V3, V5) interact between them to provide an unified image which is the essential prerequisite for consciousness of a visual environment. In addition, entrant and reentrant neural activities provide a network of recurring information that contribute to the consciousness of environmental visual scene. As for Milner and goodale, visual perception involves two paths interconnected on a recurring basis: a ventral path and a dorsal path. After receiving information from retina and geniculate body, the ventral path projects towards the lower part of the temporal cortex and leads to a perceptual representation of object, while the dorsal path projects towards the parietal posterior cortex and leads to the representation of movement of the object.

The electrophysiological studies of the olfactory system were mainly tackled by Walter Freeman. Although his investigations were conducted on the olfactory system in rabbit, Freeman affirmed that his results could be more general to explain the phenomenon of consciousness. He showed that some oscillations of brain waves have no simple frequencies: instead, they present erratic and unpredictable forms. When stimuli reach the cortical olfactory system, it occurs some spatial "patterns" of modulated amplitude whose emergence is the first step in the process of perception. These "patterns" go back then at the cortex by in- 
creasing amplitude modulation while alleviating sensory activity. In this recurring process, the cortex "sees" literally the resultant meanings, not the image of stimulated "patterns" which are not conscious. Stimuli following confirm or invalidate this first perceptive operation through new "patterns" of amplitude modulation, and so on. In this model, therefore, it is suggested that the chaotic activity of multiple recurring loops of "patterns" stimulates all the cerebral hemisphere to make a perceptual awareness.

\subsection{Thalamo-Cortical System [9]}

Neurobiologists Antonio Damasio, Rodolf Llinas, Jean-Pierre Changeux and Gerald Edelman proposed, in different models, that consciousness arises of the activity of a global neuronal workspace formed by some thalamo-cortical circuits. Such a workspace had been previously suggested by Bernard J. Baar [23].

The model developed by Damasio was mainly based on the genesis of emotion, feeling and memory. According to him, there is gradual emergence of two kinds of consciousness: the core consciousness and the extended consciousness. His model distinguishes the unconscious proto-self, the central-self of the core consciousness and the autobiographical-self of the extended consciousness. The proto-self is a coherent collection of neuronal configurations which, moment after moment, mapping the state of the physical structure of the body in its diverse dimensions: internal environment, viscera, vestibular system, musculo-skeletal framework, etc. This neuronal collection is owned by the brainstem, the hypothalamus, the basal telencephalon, and the amygdale. These neuronal configurations are implicit and unavailable to consciousness: there are potential configurations of activities. When these neuronal collections are activated by any stimulating emotional inductor, a number of consequences trigger what Damasio called "emotion". In the first step, interaction with the emotional inductor changes the mapping of neuronal configurations of the proto-self, thus creating the sens of self in the knowing act. In the second step, stimulating induction is mapped in sensory and motor structures of the proto-self. These two types of neural charts are known as charts of first order. A chart is a sheet of neurons whose points are systematically related, on the one hand, at points on sheets of receiving neurons to satisfy entries of signals and, on the other hand, at points located on other charts to satisfy reentries of signals. A second process is then added to the first: 1) The sensorimotor maps cause changes in the mapping of configurations of the body; 2) These changes are jointly re-represented in transitional second-order charts. Finally, the second-order charts can become mental images called "feelings" through recurring causality loops: they correspond to emergence of the core consciousness of central-self. The brain regions of second-order charts appear to be the superior colliculi of "ondulating" structures in the posterior part of the midbrain (also named "tectum"), the cingulate cortices, the thalamus, and some areas of the prefrontal cortex. At a higher level, we find the expanded consciousness of the autobiographical-self, which is based on organized archiving stored experiences of the central-self. These permanent files, 
successively formed by the conventional memorization and by the extensive memorization of intellectual and cultural data, can be in turn transformed into explicit images of actualities, events and data (stored in the autobiographical-self) through recurrent causality loops. At still higher level, these processes result in human language, creativity and moral consciousness.

Llinas was one of the first neuro-scientists to suggest that consciousness arises of the iterative recurrent thalamo-cortical activity. In his theory, consciousness is the result of Gamma wave oscillations of recurrent circuits which include the pyramidal layer IV of the cortex, the intralaminar non-specific cores of the cortex, and the non-specific thalamic cores. In the same vein, Jean-Pierre Changeux and Stanislas Dehaene proposed that consciousness arises of a global thalamo-cortical workspace. According to these authors, this space is made up of neurons having long axons, able to disseminate information in areas that connect the thalamic nuclei of palliothalamus with the prefrontal areas, mainly the parieto-temporal and cingulate regions. Access to consciousness results from the sudden ignition of neurons having long axons, particularly dense in the prefrontal and parietal cortex. Recent experiments revealed that consciousness emergence corresponds to late synchrony of these neurons in the Beta band (13 $30 \mathrm{~Hz}$ ), resulting in establishment of a permanent dialog between the thalamic and cortical regions. Access to consciousness so is a global, sudden and synchronous phenomenon, which implies reciprocal causation between the concerned regions through a considerable amount of cyclically recurring paths. It is based on the three major distinctions introduced by Kant in the "Critique of pure reason", about the nature and constitution of the "world" understood as the sum-total of all appearances: 1) development of representations from objects in the world; 2) abstraction of these representations into concepts; 3 ) organization of these abstractions into higher order concepts. In this model, therefore, one goes from the memorized image to concept and thought, the concept being the formalized image of the object (the prototype of the object), and the thought being a "calculation" on mental object.

In the model of Edelman, consciousness results of dynamic activities of neuronal charts in many different areas often very remote in the thalamo-cortical circuits. Consciousness emerges from a dynamic system of neuronal groups which, at some points, are synchronized and interact between them. The theory of Edelman is based on the neuronal darwinism: not only it involves a structural selection of neuronal circuits during the fetal period, but it also involves a functional adaptive selection in the adult life. Like the immune system (Edelman shared the 1972 Nobel Prize for his work on the antibody structure), brain recognition system reacts to external events by selecting each time groups of neurons. This selection requires the existence of interactions between neuronal charts, with permanent recursive exchanges of entries and re-entries. Edelman proposed that reentries between posterior, modality-specific cortical areas and more anterior areas related to memory and executive functions, provide a mechanism for conscious processes without a homunculus to do the recognizing 
for it. These long-range and massively parallel connections from one brain area to another provide the dynamic spatio-temporal coordination in neuronal groups that is necessary for integrated and adaptive conscious behavior. Reentrant activity also allows the brain area, having responses originally evoked by sensory input, to give similar responses in the absence of that input. Such a reentrant activity is a necessary process for memory. Thus, the present model helps to explain, for example, that thirty-three charts of visual perception, which are widely dispersed and functionally isolated, are capable of triggering the perceptive process that connects the edges, directions, content and movement in a coherent way to give the perceptual image of the object.

In his model, Edelman distinguished primary consciousness and secondary consciousness. Primary consciousness, named "remembered present", can be defined as simple awareness that includes perception and emotion. It refers to being mentally aware of things in the world in the present without any sense of becoming, and is composed of mental images bound to a time around the measurable present. Secondary consciousness, or awareness of consciousness, is based on the ability of the dynamic workspace core to be able to connect the areas involved in the primary consciousness to areas that mediate the semantic activity. Responses triggered by the dynamic core leave the cortex by parallel, polysynaptic unidirectional paths to reach some unconscious areas (basal ganglia and some thalamic nuclei) and then return to cortex. In this way, the dynamic core connects the memory of "category-values" and moral codes as also the conventional memory to conceptual charts. Let us recall that the memory of "category-values", which is stored during the human evolution, involves the bodily functions such as the heart rhythm, the sexual behavior, the endocrinal functions, the vegetative functions, etc. Since the dynamic core has the semantical ability to give sense (in human and some primates), it explicates the self, the past and the future by converting signals from the brain and the world in a "phenomenal transformation". The "phenomenal transformation" is the experience in which the dynamic thalamo-cortical core "speaks to itself". Unlike the animals, this ability to have mental images through recurring process allows man to be capable of setting himself free of the "remembered present". Secondary consciousness reaches its higher level when the semantical ability allows to develop a genuine linguistic capability. In sum, the theory of Edelman states that primary and secondary consciousness are based on a dynamic holistic neuronal core made up of a multitude of paths of recurring causation. Although the holism theory usually lays down that parts of a whole are in intimate interconnection, such that they cannot exist independently of the whole, the holistic process is considered in the present paper to be a cybernetic system whose activity is governed permanently by results of this activity.

\subsection{Approaches of Cognitive Reversal [22]}

In the traditional philosophical and scientific studies of consciousness, explanation of intentionality was generally based on theories of consciousness. On the 
contrary, in the recent cognitive approaches, consciousness depends on intentionality: conscious access to mental contents becomes a secondary issue compared to the study of the treatment of information. That is, there is a cognitive reversal between the problem of consciousness and the problem of intentionality. This cognitive reversal was mainly introduced by Henri Atlan from 1990. In his initiating article "Project and significance in the networks of automata", Atlan showed that within the framework of cognitive science, intentionality is a special form of causality that has the ability to achieve certain aims and to operate on some informational contents. Such a system can adapt its program when it is faced with a new field of meanings. To Atlan, the human being is thus capable of creating indefinitely the novelty. Karl Pribram also sought in the same period to renew the "mind-body" relationship within the framework of the cognitive reversal. From researchs of localization of cognitive functions in brain, Pribram distinguished three modules of consciousness. The first module, related to our voluntary actions, includes a twofold pathway: a pathway located at the level of vegetative regulation systems, which concerns the involuntary processes of retroactive controls (feedback) of intentions; and a pathway located at the cerebellum level, which uses proactive controls (feedforward) allowing to calculate and to fit in advance the prehensions and actions of objects. The second module, located at the level of the sensory-motor cortex, concerns the awareness of object. It is constituted by the projection of stimuli from the sensory and muscular surfaces towards the external environment where action takes place. In the third module, at the frontal cortex level, conscious attention results of information processing system that lets the narrative consciousness get a flexible treatment of usual behaviors. In sum, in the cognitivist approach of Pribram, consciousness becomes the property of some functional neuronal circuits to possess the power of "self-scanning".

In this context of cognitive reversal, several researchers such as Alain Berthoz, Vilayanur Ramachandran and Andy Clark, showed that human cognition is able to define its own objective and create its own field of meanings. To Berthoz, cognitive systems build partial representations of environment by using one of four following spatial reference systems: 1) retino-tropic system, which allows to locate the objects compared to the position of eyes; 2 ) vestibular system of the inner ear, which allows to locate the objects compared to three axes of movements of the head; 3) postural system which locates the objects compared to vertical axe of the body; 4) some members of the body, such as hands, feet, etc. To Ramachandran, the visual system can use an algorithm calculated from the constraints observed in the living environment, and build thus a useful representation of the environmental scene by combining partial information. To Clark, adaptation of the cognitive system can be obtained by multiple feedback loops allowing a robot, for example, to recalculate the movement necessary to success of the operation. The cognitive reversal between consciousness and intentionality, therefore, leads to a richer model in which human permanently possesses a cognitive self-control and operates thus in a field open of meanings. 


\section{Essential Characteristics of Emerging Consciousness}

As seen before, most neurobiologist and neurocognitivist studies, whatever the physiological functional systems chosen, suggest all in fine that emergence of consciousness boils down essentially to a process of recurring causation. Despite the diversity of selected approaches, obtaining such a common conclusion is not surprising since each conscious experience is a whole regardless of its physiological origin-awareness of pain, for example, is the only conscious experience which invades the mind when the pain occurs. These emergentist approaches are generally presented as being a form of crypto-monism. Can we say that monistic conceiving is superior to any dualistic conceiving to allow a rational description of consciousness as unitary noetico-neuronal phenomenon? It seems that no, because it is as difficult, with the classical inducto-deductive thought, to explain the relationship that links the neuronal and noetic activities with the monistic conceiving as with the dualistic one. Monistic conceiving is here only verbal, because it assumes implicitly, as in the philosophy of Spinoza, that thought (here the consciousness) is a different attribute of body (here the neuronal material [24]). In fact, given the great diversity of results of various emergentist works, there are four possible conditions to achieve the statement of essential characters of emergentist consciousness: 1) to look for a generic definition that subsumes under it the various results; 2) to put a priori between bracket (to make an "epoche") the intimate subjective experiences of consciousness (i.e., qualia); 3) to make that noetic and neuronal activities are sui generis in the integrity of their unitary process; 4) to make that these activities are normative in a holistic space in order to have a teleologic operative identity. Such conditions should lead to a teleologic mechanism not only because of its holistic property, but also because the relationship of the system with world, due to cognitive reversal, is integrated into the cognitive operation.

To throw light on the noetico-neuronal identity of consciousness, a possible strategy is to use the cybernetic method of the philosopher Gilbert Simondon [25] [26] [27] $]^{3}$. According to Simondon, cybernetics can be called "organology",

${ }^{3}$ The project of Gilbert Simondon is to think the individual through the individuation in all areas of reality: physical, biological, psycho-social and trans-individual. It is for him to reverse the ontological privilege granted by metaphysics to being on the becoming, the result on operation, the individual on individuation, and makes thus the condition of any comprehensive knowledge of reality. Unlike the substantialism, this approach is based on the becoming of the object and subject. It assumes that the genesis of thought may be accomplished at the same time that the genesis of object. As a substitute for the classical identity of Being, Simondon suggests the concept of transductive unity, because he thinks that Being can be constantly maintained out of phase compared to itself. The transductive unity is the property that gives an account of metastability. Analogical knowledge, in the transduction process, established a relationship between the events existing out of thought and the operations of thought itself. Analogy is always co-individuation. Therefore, in what he called his "allagmatic" theory (or theory of operations), Simondon endeavours to seize the union both of the structure of Being and its holistic operation. In this context, the cybernetic method studies operation whereby some initial structure is transformed into another structure by its own functioning. This method is the Cogito of a new reflexive philosophy where the function enters itself in its operation. Since value, according to Simondon, is what for a holistic system is able to bring up a new structure of compatibility, the cybernetic method, in its balanced processes between Being and acting and between operation and structure, is a new axiontology. 
because it is the situation where Being becomes causa sui by recurrence of causality. The cybernetic method raises the event causa sui in a holistic system: it is not substantialist and requires an epistemological and ontological realism extremely different from the analytical scientism and positivism. In the cybernetic theory, the recurrence of causality involves three types of causes: the energetic cause, or efficient cause, which is the informational provisionment of the system; the structural cause, conditioned by the internal modulating structure of the effector; and the formal cause of the recurring signal. In this method, knowledge establishes a relationship between the objective activities existing out of thought and the activities of the thought itself, which operationally brings being and thought into a co-determinate relationship. That is, there is a transductive and immanent "co-individuation" of thought and of outside organic matter according to analogical principle. In this view, individuation of thought is able to accompany the individuation of matter things, because Being of subject and Being of object arise from a "more primitive reality". To Simondon, transduction and analogy constitute the theory of operations, the operation being by definition the conversion of one structure into another structure. Such a definition implies that consciousness is a process, not a substance. If we adopt this thesis, we must not search for any more a classical inducto-deductive relation between a noetic "substrate" and a neuronal "substrate", but a normative transduction between a noetic process and a neuronal process in a holistic space, that is a relation of relation. To Simondon, act is in itself a statement of implicit normativity which meets the normativity previously creates by other acts. In sum, the goal is to have a perfect epistemologic and ontogenetic merge between the noetic activity, neuronal activity and the holistic space. Thus, the relation of relation is not only logical, it becomes also a transductive process causa sui which is while becoming in a permanent search of innovation.

Since the simondian transduction is a mode of unity through the ongoing transformations of a creative act that performs an ultimate point already contained in its essential being, the emerging consciousness can be essentially boiled down in the concept of intentional Fiat. In this re-investigation of objective characters of consciousness, transduction becomes the fundamental processual meaning of intentional Fiat. By its transductive simondian nature, consciousness is a process that is more-than-unity and more-than-identity: it is constitutively non identity to itself. It is an identity which knows itself and feels itself in the same movement that it evolves unceasingly. It is neither transparent to oneself in the sense of the Cartesian Cogito, or founded in the reality which it would aim (as in the philosophy of Husserl). Consciousness may be defined only by the operational process that constitutes it. Consciousness is a reserve of becoming which is in excess of itself, somehow as a system that has a potential energy to be permanently transformed. It is a process causa sui that tries to reach permanently a pre-ontic future, which resolves itself immediately in ontic present that becomes past (memory). In other terms, consciousness cannot be assimilated with the appearance of actualities that result of choices in the field of cognitive 
meanings, that is of the reduction of uncertainty from the infinite number of possibilities. Conceptually, consciousness is always ahead of itself because it is not the choice that is made but the choice that is anticipated. Let us specify that this permanent research of becoming is not about a simple actualization of possibilities predetermined by an ultimate future point. Becoming is not thought any more here as in the ontology of Greeks, because it is rather about an advent which is referred to nothing, as absolute as being. Research of future is a question that remains permanently "wanted". By proposing that intentional Fiat of consciousness is a permanent quest for an unattainable future, this model is placed in the perspective of a creative act that needs to internalize oneself constantly, unable to escape ontological anthropogenic limits of the finitude of human beings. Nevertheless, in this back on itself by which it rebuilds unceasingly a world (a "mondeity"), intentional Fiat made of consciousness an eminent exercise of freedom insofar as it is a constant research for novelty in the open field of possibilities (or of cognitive meanings acquire through the cognitive reversal).

\section{Essential Characteristics of the Homeostasis}

Homeostasis is usually considered as the self-regulating dynamic process that supports the survival of every living being. It results of the coexistence and complex articulations of infinity of recurring molecular activities, entangled in a hierarchy of different organization levels. Hypothesis of Mainzel about the nonlinear dynamical systems of feedback loops should therefore be a useful approach for studying this topic. A considerable amount of studies have shown that molecules involved in intra and extracellular signaling are made up, not by linear pathways as one thought it in the past, but by complex networks in which are orchestrated myriads of molecules having for most enzyme activities [28] [29] [30]. These networks have the following properties: 1) They are self-regulated with various feedback loops whose the role is to manage the homeostasic process of the system; 2) They operate on space-time by several orders of magnitude scales so that the signalings may be autocrine, paracrine or endocrine; 3) They are usually connected between them by molecular binding "nodes" (which are often kinasic enzymes); 4) They are dependent on mechanisms managed by cytoskeleton, by sub-cellular organelles (for example, mitochondrial organelles), and by vesicular traffics [31]. Moreover, not only they are connected to complex networks of metabolisms, but they are also connected to genome-regulating systems. The actual trend in the new so-called "post-genomics" biology is indeed to admit that epigenetic networks can control certain genes, thus reversing the causal molecular chain which appeared so far undisputed [28] [32] [33]. According to Henri Atlan, for example, program resides in the whole of cellular organisms (including cytoplasm), genome becoming only a "data bank" that feeds the cellular program [34]. All signaling networks, metabolic paths and genome-regulating epigenetic paths of cells constitute thus self-regulating molecular hyper-networks of cybernetic functioning, whose organization is self-individualized thanks to dynamic states of the operational cell membrane. In brief, the ho- 
meostasic system of living beings can be metaphorically described as a generic pathway of cybernetic recurring causation, which subsumes under it the myriad of molecular feedback loops of organism.

It is admitted that a homeostasic system keeps to a permanent tension between the local and global organizations, which are regulated within the limits imposed by structurally stable attractors of integration. These organizations have several types of temporality: they can be cyclical, stable, chaotic or chaotico-cyclical. Three categories of constraints at least appear to condition their dynamism [35] [36]. The first constraint is the maintenance of critical perdurante area in a dynamical phase space which comes from the convergence of two opposing phenomena: on the one hand, some critical local bifurcations which tend to be deviated from the balanced average situation; one the other hand, the overall self-organizing stability of all states. The second constraint results from the autopoietic nature of complex network of both local and global relations, which unceasingly deconstruct and rebuild themselves to maintain a topological unity with respect to environmental adversity. The third constraint results from the fact that it is need to have, facing to environment, the self-maintaining of an internal order in the different local and global organizations. These homeostatic constraints promote the best thermodynamic conditions for self-maintaining of living beings. Let us look at what these conditions could be.

\section{Thermodynamic Conditions for Self-Maintaining}

Thermodynamics generally describes energy exchange processes of macroscopic systems. Objects as varied as liquids, magnets, superconductors, living things, and even black holes, comply with its laws. In macroscopic systems, behavior is reproductible and fluctuations (deviations from the typically observed) are small. However, as dimensions of systems decrease, fluctuations away from equilibrium begin to dominate their behavior: in a nonequilibrium small system, thermal fluctuations can lead to significant deviations from their average behavior [37]. In parallel to development of experimental investigations to study properties of small systems, therefore, physicists developed several theories collectively known as fluctuation theorems, Jarzynski equality and Crooks theorem. Let us begin by supposing that each recurring path of the above described hyper-network is a small thermodynamic system in a thermal nonequilibrium state that obeys to the Crooks theorem [38]. Consider also that this small system, kept in contact with an external bath at temperature $T$, has its nonequilibrium state determined by a control parameter $\mathrm{x}$. Initially, the control parameter is $x(0)$ and the system is in equilibrium state. The nonequilibrium process is obtained by changing $x$ to obtain the final value $x(t)$. Imagine that we let time interval pass while keeping our system at $T$ temperature. The Crooks theorem [38] then asserts that, in a heat bath-coupled time-symmetrically driven nonequilibrium system, any trajectory starting at $x(0)$ and going through microstates $x(t)$ obeys to the relation: 


$$
\ln \left|\frac{p[x(t)]}{p[\tau-t]}\right|=\frac{\Delta Q}{T}
$$

where $p[x(t)]$ and $p[\tau-t]$ stand for the probability distribution along the forward trajectory $x(t)$ and the reversed one $x[\tau-t]$ respectively, $\Delta Q$ being the heat released into the bath over the course of $x(t)$. By fixing the starting and ending points of our trajectory $((x(0))=i, x(\tau)=j)$, we can average the exponential weight of the forward heat over all paths from $i$ to $j$ and obtain:

$$
\frac{\pi(j \rightarrow i ; \tau)}{\pi(i \rightarrow j ; \tau)}=\left\langle\mathrm{e}^{\frac{-\Delta Q_{i \rightarrow j}^{\tau}}{T}}\right\rangle i \rightarrow j
$$

The transition matrix $\pi(i \rightarrow j ; \tau)$ is the conditional probability that the macroscopic system is found to be in state $j$ at time $t=\tau>0$. This relation allows to formalize the myriad of small systems of recurring causation in terms of alive entity. Let us suppose now that probability distributions $P(i \mid A)$ and $P(j \mid B)$ are the probabilities that the system is successively in macrostates $A$ and $B$ (the transition $A \rightarrow B$ is supposed to be very small). As described by Gong and Quan [39], we can then define associated quantities that will give a definition of the irreversibility of the system by writing the following equations:

$$
\begin{aligned}
& \pi(A \rightarrow B)=\int_{B} \mathrm{~d} j \int_{A} \mathrm{~d} i P(i \mid A) \pi(i \rightarrow j) \\
& \pi(B \rightarrow A)=\int_{A} \mathrm{~d} j \int_{B} \mathrm{~d} i P(j \mid B) \pi(j \rightarrow i)
\end{aligned}
$$

The first equation gives the likelihood that the system will be observed to satisfy B after time $\tau$. The second equation gives the likelihood that, after another interval $\tau$, the system will be observed again to satisfy $A$. Taking the ratio of these two quantities:

$$
\begin{aligned}
\frac{\pi(B \rightarrow A)}{\pi(A \rightarrow B)} & =\frac{\int_{A} \mathrm{~d} i \int_{B} \mathrm{~d} j\left[\frac{P(j \mid B)}{P(i \mid A)} P(i \mid A) \pi(j \rightarrow i)\right]}{\int_{A} \mathrm{~d} i \int_{B} \mathrm{~d} j[P(i \mid A) \pi(i \rightarrow j)]} \\
\frac{\pi(B \rightarrow A)}{\pi(A \rightarrow B)} & =\frac{\int_{A} \mathrm{~d} i \int_{B} \mathrm{~d} j\left[\frac{P(j \mid B)}{P(i \mid A)} P(i \mid A) \pi(j \rightarrow i)\right]}{\int_{A} \mathrm{~d} i \int_{B} \mathrm{~d} j[P(i \mid A) \pi(i \rightarrow j)]} \\
& =\left\langle\frac{\mathrm{e}^{\frac{-(\Delta Q) i \rightarrow j}{T}}}{\mathrm{e}^{\ln \frac{P(i \mid A)}{P(j \mid B)}}}\right\rangle A \rightarrow B
\end{aligned}
$$

We obtain:

$$
\left\{\left(\mathrm{e}^{\ln \frac{P(i \mid A)}{P(j \mid B)}-\ln \frac{\pi(B \rightarrow A)}{\pi(A \rightarrow B)}}\right)\left(\mathrm{e}^{\frac{-(\Delta Q) i \rightarrow j}{T}}\right) i \rightarrow j\right\}=1
$$

We arrive thus immediately at:

$$
\left[\frac{\Delta Q}{T}\right] A \rightarrow B+\ln \left[\frac{\pi(B \rightarrow A)}{\pi(A \rightarrow B)}\right]+\Delta S_{\mathrm{int}} \geq 0
$$


$\Delta S_{\text {int }}$ is the internal entropy variation when the system change from $A$ to $B$. If $A$ and $B$ correspond to identical macrostates, then we should have $\pi(B \rightarrow A)=\pi(A \rightarrow B)$ and the above relation reduces to the Second

Thermodynamic Law. In this case, the change of entropy of the bath $\left(\frac{\Delta Q}{T}\right)$ and $\Delta S_{\text {int }}$ must be greater than or equal to zero. However, since the change just described is a highly irreversible process $\left(\ln \left[\frac{\pi(B \rightarrow A)}{\pi(A \rightarrow B)}\right]\right.$ is negative), the change $\frac{\Delta Q}{T}+\Delta S_{\text {int }}$ should be the minimum positive entropy production.

With those results in mind, let us suppose that the alive entity has a population of $n \gg 1$ at $T$ temperature which obeys a master equation of the form:

$$
p_{n}(t)=\zeta n\left[p_{n-1}(t)-p_{n}(t)\right]-\xi n\left[p_{n}(t)-p_{n+1}(t)\right]
$$

where $p_{n}(t)$ is the probability of having a population of $n$ at time $t$, and $\zeta>$ $\xi>0$.

Let us introduce the behaviors in the system, i.e. activities. Whatever the exact value of $n$, the probability in the short period of time $d t$ that one particular change $A \rightarrow B$ gives $\pi(A \rightarrow B)$ is $\zeta d t$, while the probability to have $\pi(B \rightarrow A)$ is $\xi d t$. Thereby:

$$
\left[\frac{\Delta Q}{T}\right] A \rightarrow B+\Delta S_{\mathrm{int}} \geq \ln \left[\frac{\zeta}{\xi}\right]
$$

By rearranging this expression, we obtain:

$$
\zeta=\xi \mathrm{e}^{\left[\frac{\Delta Q}{T}\right] A \rightarrow B+\Delta S_{\text {int }}}
$$

Let us consider that the two different macrostates $A$ and $B$ have different dissipated heat $\Delta Q$ when $\Delta S_{\text {int }}$ and $\xi$ are fixed. If $\Delta Q>\Delta Q^{\prime}$ then clearly $\zeta>\zeta^{\prime}$ : the system that dissipates more heat has the macrostate transition faster (i.e., the more important activity). Now, it can be expected that even far from equilibrium, the Shannon entropy $\left(S_{\text {int }} \equiv \ln p\right)$ remains the measure of statistical disorder in the system that it is for equilibrium system. Thus, if one consider the case of uniform starting and ending distributions, where $p(i \mid A)=p_{I}$ and $p(i \mid B)=p_{I I}$, we can write $\Delta S_{\text {int }}=\ln \left(p_{I} / p_{I I}\right)$. The relation $\ln \left(p_{I} / p_{I I}\right)$ thus measures how many more probability there is in $B$ than in $A$ in the system far from equilibrium. Therefore, when the system is very irreversible (i.e. when the transition of two macrostates is very fast), dissipated heat is important and $\Delta S_{\text {int }}=\ln \left(p_{I} / p_{I I}\right)$ is continuously maintained in a lower value during the macrostate transition. These results suggest that if the important dissipated entropy of the bath $\left(\frac{\Delta Q}{T}\right)$ is under the influence of a driving thermodynamic process, then activity of the alive individual is high, its $\Delta S_{\text {int }}$ is continuously maintained in lower values, and an order is kept in its structure to ensure the 
self-maintaining. These results are consistent with the original idea of Jeremy England which was recently tested in computer simulations. This approach show that groups of atoms in an open system, which generally tend to adopt configurations to arrive at a state of "thermodynamic equilibrium", can behave differently when they are driven by external energy sources. In this case, they tend to start tapping those energy sources, aligning and rearranging so as to better absorb the energy and dissipate it as heat to keep their internal entropy low and adapt their ordering [40].

\section{Towards a Self-Maintaining Process Driven by the Entropy Production}

Let us show, now, that the driving process that ensures the self-maintaining of the living being may be the entropy production of the "Earth + Universe" system. In the Earth system, many processes are producing entropy. Numerous works have shown that the biotic activity of Earth generates substantial amounts of chemical free energy. The entropy gap between this generation of free energy and the energy consumption by living beings and physical engines is the free energy left in the neighbouring of the Earth to drive all activities [41] [42] [43]. Since, the produced entropy is exported into the interstellar radiation field, this could mean naively that the variation of entropy $(\mathrm{d} S / \mathrm{d} t)$ is equal to zero. Now, studies have provided a holistic description of the functioning of the whole Earth system that is grounded in the generation, transfer and dissipation of free energy. Axel Kleidon [44] [45], for example, has thoroughly studied how the Earth system generates power, how it transfers it, and how power is required to evolve thermodynamic variables away from equilibrium. In his approach, what is called "Earth system" involves the Earth and its surroundings. Theory of Kleidon states that the change of internal energy $\mathrm{d} U$ of the system is balanced by the exchange of heat $\mathrm{d} Q$ with its surroundings and the work done by the system $\mathrm{d} W: \mathrm{d} U=\mathrm{d} Q$ $-\mathrm{d} W$ (First Thermodynamic Law). Here, $\mathrm{d} W>0$ if the work is done by the system, and $\mathrm{d} W<0$ if the work is done on the system by the surroundings. Then, the theory follows the direction given by the Second Law of Thermodynamics. It states that the entropy of an isolated system can only increase with time, i.e. $\mathrm{d} S \geq$ 0 . For small changes, the change of entropy is given by: $\mathrm{d} S=\mathrm{d} Q / T$. When a small amount of heat $\mathrm{d} Q$ is removed from a hot reservoir of temperature $T_{h}$ and added to a cold reservoir of temperature $T_{c}$, the total entropy produced by transferring heat from hot to cold is: $\mathrm{d} S_{t o t}=\mathrm{d} S_{h}+\mathrm{d} S_{c}=\mathrm{d} Q\left(1 / T_{c}-1 / T_{h}\right)$. Since $T_{c}<T_{h}, \mathrm{~d} S_{\text {tot }}>0$ and the process of transferring heat is irreversible: it produces entropy, it acts to deplete the gradient $T_{h}-T_{\mathcal{c}}$ and it acts to bring the system closer to a state of thermodynamic equilibrium in which $T_{h}=T_{c}$

To study evolution to thermodynamic equilibrium, a simple model as above should consist of two reservoirs of different initial heat contents (with equal heat capacity $c$ and temperatures $T_{h}$ and $T_{c}$ ). These reservoirs exchange heat with each other but are isolated to the surroundings. Expressing the heat flux between the reservoirs as $J_{\text {heat }}=\mathrm{d} Q / \mathrm{d} t$ (with a positive flux directed from $T_{h}$ to $T_{c}$ ), we 
obtain the two differential equations that describe the temperature evolution of heat reservoirs with time: $c \cdot \mathrm{d} T_{h} / \mathrm{d} t=-J_{\text {heat }}$ and $c \cdot \mathrm{d} T_{c} / \mathrm{d} t=J_{\text {heat }}$. Hence, the respective entropies $S_{h}$ and $S_{c}$ are: $\mathrm{d} S_{h} / \mathrm{d} t=-J_{\text {heat }} / T_{h}$, and $\mathrm{d} S_{c} / \mathrm{d} t=-J_{\text {heat }} / T_{c}$. One obtains thus:

$$
\mathrm{d} S_{\text {tot }} / \mathrm{d} t=\mathrm{d} S_{c} / \mathrm{d} t+\mathrm{d} S_{h} / \mathrm{d} t=J_{\text {heat }}\left(1 / T_{c}-1 / T_{h}\right)=\sigma_{\text {heat }}
$$

so that the increase of the total energy of two reservoirs equals the total amount of entropy produced $\left(\sigma_{\text {heat }}\right)$ by the heat exchange process within the system. This shows how the initial temperature gradient $T_{h}-T_{c}$ is depleted by the flux $J_{\text {heat }}$ in time. The heat flux $J_{\text {heat }}$ depletes the entropy of the hot reservoir and, by adding heat to the cold reservoir, the entropy is increased in this reservoir. Due to the mixing of heat contents, this increase of entropy in the cold reservoir is greater than the reduction of entropy in the hot reservoir. Hence, the overall entropy $S_{t o t}$ increases with time.

To explain how entropy exchange across the system boundary can be maintained away from an equilibrium state of thermodynamic, we need to alter the energy balances of the model considered before to:

$$
c \cdot \mathrm{d} T_{h} / \mathrm{d} t=J_{\text {in }, h}-J_{\text {out }, h}-J_{\text {heat }}, c \cdot \mathrm{d} T_{c} / \mathrm{d} t=J_{\text {in }, c}-J_{\text {out }, c}+J_{\text {heat }}
$$

and

$$
\begin{aligned}
\mathrm{d} S_{h} / \mathrm{d} t & =J_{\text {in }, h} / T_{\text {in }}-J_{\text {out }, h} / T_{h}-J_{\text {heat }} / T_{h}+\sigma_{\text {mix }, h} \\
\mathrm{~d} S_{c} / \mathrm{d} t & =J_{\text {in }, c} / T_{\text {in }}-J_{\text {out }, c} / T_{c}+J_{\text {heat }} / T_{c}+\sigma_{\text {mix }, c}
\end{aligned}
$$

where the entropy associated with the incoming energy is associated with the temperature $T_{i n}$. Entropy is produced by the mixing of the incoming energy fluxes $J_{i n, h}$ and $J_{i n, c}$ in the two reservoirs at temperatures $T_{h}$ and $T_{c}$. We therefore obtain:

$$
\sigma_{m i x, h}=J_{i n, h}\left(1 / T_{h}-1 / T_{i n}\right) \text { and } \sigma_{m i x, c}=J_{i n, c}\left(1 / T_{c}-1 / T_{i n}\right)
$$

and by mixing associated with heat flux $J_{\text {heat }}$ from the warm reservoir to cold reservoir:

$$
\sigma_{\text {heat }}=J_{\text {heat }}\left(1 / T_{c}-1 / T_{h}\right)
$$

Finally, the entropy budget of the whole system is given by the following equation:

$$
\mathrm{d} S_{\text {tot }} / \mathrm{d} t=\mathrm{d} S_{h} / \mathrm{d} t+\mathrm{d} S_{c} / \mathrm{d} t=\sigma_{m i x, h}+\sigma_{m i x, c}+\sigma_{\text {heat }}-L
$$

where $L=\left(J_{\text {out }, h} / T_{h}+J_{\text {out }, c} / T_{c}\right)-\left(J_{i n, h}+J_{\text {in }, c} / T_{\text {in }}\right)$ is the net entropy exchange across the system boundary. Consequently, the initial temperature gradient is depleted as previous, but it does not vanish in the steady state. Instead, a heat flux transports heat from warm to cold to deplete the temperature gradient that is continuously built by the differential heating $J_{i n, h}-J_{i n, c}$ This heat flux produces entropy by the depletion of the temperature gradient, but instead of increasing the entropy of the system to the maximum, the produced entropy in steady state is exported by the enhanced export of entropy associated with the outgoing heat flux $J_{\text {out }, h}+J_{\text {out }, c}$ In sum, the theory of Kleidon shows that in the Earth system, 
there is an unceasing maintain of entropy exchange away from the thermodynamic equilibrium state which results from the overall lower temperature at which the total amount of received heat is exported to the surroundings.

Although the current entropy content of the Universe is also a complex question, it is simpler to deal with because the boundaries of the Universe are not an issue. Most cosmologists make assumption that entropy of the Universe is increasing since the hot Big Bang 13.8 billions years ago [46] [47]. Furthermore, there is much evidence that the Universe is homogeneous on scales above about 100 million light years. This homogeneity makes the distinction between the very large control volume (100 million light years) of Universe and its environment meaningless. This volume is so large that the average density of black holes, supernovae, stars and planets, accurately represents the average density everywhere in the Universe. It follows that we can ignore the boundary problems and write that $\mathrm{d} S / \mathrm{d} t>0$ everywhere in Universe. Based on the entropy budgets of the Eart and the Universe, therefore, it can be proposed that dissipated heat, activities and $\Delta S_{\text {int }}$ by the organism could be driven by the production of entropy of the entire "Earth + Universe" system.

\section{Towards a Model of Intentional Embodied Consciousness}

Psychologists and philosophers both investigate the nature of the mind from rather different angles. Psychologists and neuro-scientifists study the actual mechanisms in brain and body which underpin mental events and processes. By contrast, philosophers ask more abstract questions: for example, about how can be designed the relationship between the noetic activity and the subjacent neuronal activity. However, psychology and philosophy are not opposed disciplines and are complementary. Although many scientists do not deny there is a difficult mind-body problem, they generally admit that individuals are physiological systems with mental and material properties, and intentionality of consciousness belongs both to brain and body [4]. According to the embodiment thesis, many features of cognition are embodied in that they are deeply dependent upon characteristics of the physical body of the individual, such that the mind-body intricacy plays a significant causal role, or a physically constitutive role, in the intentional cognitive processing of individual [5] [6] [21]. In the present article, we argue that it is not wrong to think this. For the entropy of the "Earth + Universe" system is to be equated with the pole objet, it is necessary that the entire "brain + body" is equated with the cognitive pole, i.e. have an intentional Fiat of consciousness that is stretched to the future of this object. Therefore, it is necessary that the intentional noetico-neuronal unit is extended to entire physiological system "brain + body". In other words, the whole "brain + body" should have an intentional Fiat of embodied consciousness. Let us show how such a hypothesis is possible.

As argued by Andrieu [1], there is a difference between the disposed body and the intentional mental. The disposed body occurs before the intentional mental. 
The disposed body produces contents of action in the disposed mind unless there is a nomologic determinism of the first in the second. The disposed mind receives information of the disposed body without being aware of it, thinking even to determine per se the contents of its will. Following the worldly incorporation, the disposed body gives thus a neurofonctional structure to brain. The brain then transforms this provision in "intentionant" by the production, conservation and dynamic adaptation of neuronal networks in charts and behaviors. It imposes innate structures, recurring diagrams and neurocognitive adaptations to requirements of action. "Intentionant", unlike the intentional mental, is in dynamic interaction with its neuronal structure made up and activated by confronting the brain with the heterogeneity of worldly information. The transition from "intentionant" to intentional mental is carried out by the noetic activity of brain. Thus, the variations of intensity and nature of the worldly thing are built in the brain by making singular its neuronal structure for each individual. The brain is "corporant" by the indefinite incorporation of information which defines somato-sensory and somato-motrice charts. Consequently, the outside of the body is a world directed for action which gives to body a cerebral sense by making of it its environment. The continual adaptability of this cerebral sense facilities inscription of the world within brain. It results that the physiological unity of living beings is a whole [1]. This whole is not only more than the sum of its parts, it is "one" in each part and "one" outside of unity of its multiformity. A physiological system is at the same time a condition ensuring the connection of the multiformity of all elements, and a unity beside this unity of the multiformity (subsisting under itself). It is indeed the entire physiological system (brain + body) which makes this experience, since it is its parts the connection of the unity for oneself and of the unity in the multiformity. The brain thus represents the whole while being a part of the whole. The intentional Fiat of consciousness thus results from the close interaction with the body as a participant in the act to carry out. Specifically, the intentional response and the activity of the body are therefore dependent on one of the other. By bodily activity we hear only in this model either a short trip or a small change of state. Activities such as development, differentiation, cellular replication, division, etc, are of a too bewildering complexity so that they can be treated with the present simplified thermodynamic approach.

It results from the long preceding series of data and arguments that intentional Fiat of embodied consciousness could be the unitary response of the whole to increasing production of entropy of the "Earth + Universe" system, to maintain low values of the internal entropy of living beings (an order in their structures) and thus ensure their self-maintaining. Being who lives would have the intentional Fiat of embodied consciousness for ground of his entity, but such ground, not consciously, would be itself supported by the increasing production of the entropy of the "Earth + Universe" system. It is this continuous activity that should characterize the being who lives, pulled towards the unattainable future by a fatal increasing entropy of cosmological order. This activity of the being 
should maintain both his intentional embodied consciousness and his individual entity. To do this, the being should absorb the energy and dissipates it as heat to keep his entropy low and an order in his structure by increasing the entropy of its surroundings. The production of entropy of the pole object would be thus causally determinative for the production of entropy of the pole subject and causally determined by this last: pole object and pole subject would be linked by a circular causation.

\section{Conclusion}

We show here that: 1) the emerging consciousness appears to be a generic recurring process which subsumes the various results of scientist approaches under it; 2) homeostasis can be also metaphorically described as a generic recurring process which subsumes the myriad molecular feedback loops of organism under it. Based on a very large number of data and arguments, these noetico-neuronal and self-regulating homeostasic processes may be viewed as forming a fully unitary physiological system (brain + body) that is continuously driven by the fatal increasing cosmological entropy. It is proposed that this increasing entropy keeps internal entropy low and an order in the organism to allow the unceasing activity of the individual. It is this activity, pulled towards an unattainable future, that would maintain both intentional embodied consciousness and individual entity of the being that lives.

\section{Conflicts of Interest}

The author declares no conflicts of interest regarding the publication of this paper.

\section{References}

[1] Andrieu, B. (2010) Le vécu corporel du cerveau propre. Revue Philosophique de Louvain, 3, 1-18.

[2] Berthoz, A. and Petit, J.-L. (2003) Nouvelles propositions pour une physiologie de l'action. Intellecta, 36-37, 367-372.

[3] Berthoz, A. and Petit, J.-L. (2006) Phénoménologie et physiologie de l'action. Odile Jacob, Paris.

[4] Crane, T. (2007) Introduction: The Mental and the Physical. Translated by Marcus Wild and Simone Ungerer, Fischer Verlag, Frankfurt. https://doi.org/10.1017/CBO9780511611346.001

[5] Edelman, G.M. (2006) Embodiment of Mind. Daedalus, 135, 23-32. https://doi.org/10.1162/daed.2006.135.3.23

[6] Wilson, R.A. and Foglia, L. (2017) Embodied Cognition. The Stanford Encyclopedia of Philosophy, Spring Edition, Edward N. Zalta (Ed). https://plato.stanford.edu/archives/spr2017/entries/embodied-cognition/

[7] Blanquet, P.R. (2015) New Issue to Modeling Intentionality in the Field of Consciousness. Journal of Behavioral and Brain Sciences, 5, 348-367. https://doi.org/10.4236/jbbs.2015.59035

[8] Nicolis, G. and Prigogine, J. (1989) A la rencontre du complexe. Presse Universitaire 
de France, Paris.

[9] Blanquet, P.R. (2011) Advances in Interdisciplinary Researches to Construct a Theory of Consciousness. Journal of Behavioral and Brain Sciences, 1, 242-261. https://doi.org/10.4236/jbbs.2011.14031

[10] Stengers, I. (2002) Penser avec Whitehead. Eds du Seuil, Paris.

[11] Deleuze, G. (1983) Cours à l'Université de Vincennes, Paris (22/3/83-12/4/83).

[12] Ottaviani, D. (2008) La métaphysique de la lumière au moyen âge. In Philosophie de l'humanisme, Centre d'études en rhétorique, Philosophie et histoire des idées. http://www.cerphi.net/hum/lumcours1.htm

[13] Davidson, D. (2001) Subjective, Intersubjective and Objective. Oxford University Press, UK. https://doi.org/10.1093/0198237537.001.0001

[14] Merleau-Ponty, M. (1945) Phénoménologie de la perception. Eds Gallimard, Paris.

[15] Dastur, F. (1984) Consciousness and Body in the Phenomenology of Merleau-Ponty: Some Remarks Concerning Flesh, Vision, and World in the Late Philosophy of Maurice Merleau-Ponty. In: Tymieniecka, A.-T., Ed., Phenomenology of Life in a Dialogue between Chinese and Occidental Philosophy, Springer International Publishing, Berlin, Heidelberg, 117-125. https://doi.org/10.1007/978-94-009-6262-0_9

[16] Thompson, E. and Varela, F. (2001) Radical Embodiment: Neural Dynamics and Consciousness. Trends in Cognitive Sciences, 5, 418-425. https://doi.org/10.1016/S1364-6613(00)01750-2

[17] Edelman, G.M. (2003) Naturalizing Consciousness. A Theoretical Framework. Proceedings of the National Academy of Sciences, 100, 5520-5524. https://doi.org/10.1073/pnas.0931349100

[18] Mainzer, K. (2009) Challenges of Complexity in the $21^{\text {st }}$ Century. An Interdisciplinary Introduction. European Review, 17, 219-236. https://doi.org/10.1017/S1062798709000714

[19] Mainzer, K. (2009) From Embodied Mind to Embodied Robotics: Humanities and System Theoretical Aspects. Journal of Physiology, 103, 296-304. https://doi.org/10.1016/j.jphysparis.2009.08.012

[20] Damasio, A. (1999) The Feeling of What Happen. Body and Emotion in the Making of Consciousness. Harcourt Brace \& Company, New York.

[21] Leitan, N.D. and Murray, G. (2014) The Mind-Body Relationship in Psychotherapy: Grounded Cognition as an Explanatory Framework. Frontiers in Psychology, 5, 472-484. https://doi.org/10.3389/fpsyg.2014.00472

[22] Dedeurwaerdere, T. (2000) Le renversement cognitiviste et les theories de la conscience. Revue Philosophique de Louvain, 98, 732-760. https://doi.org/10.2143/RPL.98.4.542008

[23] Baars, B.J. (1988) A Cognitive Theory of Consciousness. Cambridge University Press, Cambridge.

[24] Appuhn, Ch. (1965) Euvre, Eds Garnier. Vol. 4, Trans. Opera, S., Gebhardt (ed.), Heidelberg, 1972.

[25] Simondon, G. (1998) L’individu et sa genèse physico-biologique. Eds Jérome Million (Collection Krisis), Paris.

[26] Combes, M. (2012) Gilbert Simondon and the Philosophy of the Transindividual (La Marre, T., Tr.). MIT Press, Cambridge.

[27] Simondon, G. (2016) Sur la philosophie 1950-1980. Presses Universitaires de 
France, Paris.

[28] Papin, J.A., Hunter, T., Palsson, B.O. and Subramaniam, S. (2005) Reconstruction of Cellular Signaling Networks and Analysis of Their Properties. Nature Reviews Molecular Cell Biology, 6, 99-111. https://doi.org/10.1038/nrm1570

[29] Klipp, E. and Liebermeister, W. (2006) Mathematical Modeling of Intracellular Signaling Pathways. BMC Neuroscience, No. 1, S10. https://doi.org/10.1186/1471-2202-7-S1-S10

[30] Nussinov, R. (2013) The Spatial Structure of Cell Signaling Systems. Physical Biology, 10. https://doi.org/10.1088/1478-3975/10/4/045004

[31] Mordret, G. (1993) MAP Kinase: A Node Connecting Multiple Pathways. Biology of the Cell, 79, 193-207. https://doi.org/10.1016/0248-4900(93)90138-5

[32] Vecsey, C.G., Hawk, J.D., Lattal, K.M., Stein, J.M., Fabian, S.A., Attner, M.A., Cabrera, S.M. and McDonough, C.B. (2007) Histone Deacetylase Inhibitors Enhance Memory and Synaptic Plasticity via CREB: CBP-Dependent Transcriptional Activation. Journal of Neuroscience, 27, 6128-6140. https://doi.org/10.1523/JNEUROSCI.0296-07.2007

[33] Blanquet, P.R., Mariani, J. and Fournier, B. (2009) Temporal Assessment of Histone H3 Phospho-Acetylation and Casein Kinase 2 Activation in Dentate Gyrus from Ischemic Rats. Brain Research, 1302, 10-20.

https://doi.org/10.1016/j.brainres.2009.09.030

[34] Atlan, H. (2011) Le Vivant Post-Génomique ou qu'est-ce que l'auto-organisation? Eds Odile Jacob, Paris.

[35] Bailly, F. and Longo, G. (2006) Mathématique et science de la nature. La singularité physique du vivant, Eds Hermann, Paris.

[36] Bailly, F. and Longo, G. (2008) Extended Critical Situations. Journal of Biological Systems, 165, 309-336. https://doi.org/10.1142/S0218339008002514

[37] Bustamante, C., Liphardt, J. and Ritort, F. (2005) The Nonequilibrium Thermodynamics of Small Systems. Physics Today, 58, 43-48. https://doi.org/10.1063/1.2012462

[38] Crooks, G.E. (1999) Entropy Production Fluctuation Theorem and the Nonequilibrium Work Relation for Free Energy Differences. Physical Review E, 60, 2721. https://doi.org/10.1103/PhysRevE.60.2721

[39] Gong, Z. and Quan, H.T. (2015) Jarzynski Equality, Crooks Fluctuation Theorem and the Fluctuation Theorems of Heat for Arbitrary Initial States.

[40] Horowitz, J.M. and England, J.L. (2018) Spontaneous Fine-Tuning to Environment in Many-Species Chemical Reaction Networks. Proceedings of the National Academy of Sciences, 114, 7565-7570. https://doi.org/10.1073/pnas.1700617114

[41] Dewar, R.C. (2003) Information Theory Explanation of the Fluctuation Theorem, Maximum Entropy Production, and Self-Organized Critically in Non-Equilibrium Stationary States. Journal of Physics A, 36, 631-641. https://doi.org/10.1088/0305-4470/36/3/303

[42] Dewar, R.C. (2005) Maximum Entropy Production and Non-Equilibrium Statistical Mechanics. In: Kleidon, A. and Lorenz, R.D., Eds., Non-Equilibrium Thermodynamics and the Production of Entropy: Life, Earth, and Beyond, Springer, Heidelberg, 41-56. https://doi.org/10.1007/11672906_4

[43] Martyushev, L.M. and Seleznev, V.D. (2006) Maximum Entropy Production Principle in Physics, Chemistry, and Biology. Physics Reports, 426, 1-45. https://doi.org/10.1016/j.physrep.2005.12.001

[44] Kleidon, A. (2010) Life, Hierarchy, and the Thermodynamic Machinery of Planet 
Earth. Physics of Life Reviews, 7, 424-460.

https://doi.org/10.1016/j.plrev.2010.10.002

[45] Kleidon, A. (2012) How Does the Earth System Generate and Maintain Thermodynamic Disequilibrium and What Does It Imply for the Future of the Planet? Philosophical Transactions of the Royal Society A, 370, 1012-1040.

https://doi.org/10.1098/rsta.2011.0316

[46] Lineweaver, C.H. (2014) The Entropy of the Universe and the Maximum Entropy Production Principle. In: Dewar, R.C., Ed., Beyond the Second Law, Understanding Complex Systems, Springer-Verlag, Berlin, Heidelberg, 415-427.

https://doi.org/10.1007/978-3-642-40154-1_22

[47] Liu, N., Goold, J., Fuentes, I., Vedral, V., Modi, K. and Bruschi, D.E. (2016) Quantum Thermodynamics for a Model of an Expanding Universe. 